\title{
ON THE DISTRIBUTION OF PRIME NUMBERS OF THE FORM $\left[n^{c}\right]$
}

\author{
A. $\mathrm{KUMCHEV}^{\dagger}$ \\ Department of Mathematics, University of South Carolina, Columbia, SC29208, USA \\ e-mail: koumtche@math.sc.edu
}

(Received 25 March, 1997)

Abstract. Let $\pi_{c}(x)$ be the number of the integers $n \leq x$ such that $\left[n^{c}\right]$ is prime. We shall prove that

$$
\pi_{c}(x) \gg \frac{x}{\log x}
$$

for $1<c<45 / 38$. This improves the former range $1<c<13 / 11$.

1. Introduction. The problem of representing of primes by irreducible polynomials is one of the most important problems of multiplicative number theory. However, while this problem is completely solved for linear polynomials, it is not known if there exists any polynomial of degree $n \geq 2$ that takes infinitely many prime values. There is therefore some interest in studying if there exists any "polynomial of degree $1<c<2$ " with this property. In 1953 I. I. Piatetski-Shapiro [16] showed that this is true if $c$ is not much greater than 1 . Let $\pi_{c}(x)$ be the number of the integers $n \leq x$ for which $\left[n^{c}\right]$ is prime (here $[\theta]$ is the integral part of $\theta$ ). Piatetski-Shapiro's result states that

$$
\pi_{c}(x) \sim \frac{x}{c \log x}, \quad x \rightarrow \infty
$$

if

$$
1<c<12 / 11=1.0909 \ldots
$$

Afterwards, using the fact that the upper bound for $c$ is closely connected with the estimate of an exponential sum over primes, a number of authors improved this result obtaining longer ranges for $c$. The first were G. A. Kolesnik [10] and D. Leitmann [12] who proved that (1.1) holds for any fixed real $c$ in the ranges

$$
1<c<10 / 9=1.1111 \ldots \text { and } 1<c<69 / 62=1.1129 \ldots
$$

correspondingly. In 1983 D. R. Heath-Brown [6] made two important innovations which let him obtain the range

$$
1<c<755 / 662=1.1404 \ldots
$$

which G. A. Kolesnik [11] improved again to

${ }^{\dagger}$ Research supported by Bulgarian National Science Fund - grant MM 430/94. Research done in partial fulfilment of the Ph.D requirement at the University of South Carolina. 


$$
1<c<39 / 34=1.1470 \ldots \text {. }
$$

Then, in 1990, H.-Q. Liu and J. Rivat [15] used the method of E. Fouvry and H. Iwaniec [2] for the estimation of exponential sums and proved that (1.1) holds for

$$
1<c<15 / 13=1.1538 \ldots
$$

and in his doctorial thesis J. Rivat [17] succeeded to obtain the slightly better result

$$
1<c<6121 / 5302=1.1544 \ldots .
$$

We also mention that D. Leitmann and D. Wolke [13] proved that (1.1) holds for almost all real $c \in(1,2)$ (in the sense of Lebesgue measure).

J. Rivat [17] was also the first who considered the problem for obtaining a lower bound for $\pi_{c}(x)$. Using a sieve method he proved that there exists an absolute constant $\rho_{0}>0$ such that

$$
\pi_{c}(x) \geq \rho_{0} \frac{x}{c \log x}
$$

for each fixed $c$ in the range

$$
1<c<7 / 6=1.1666 \ldots .
$$

After that R. C. Baker, G. Harman and J. Rivat [1] and C.-H. Jia [7], using the sieve method from [4] and the exponential sum estimates of E. Fouvry and H. Iwaniec from [2], independently proved (1.2) for

$$
1<c<20 / 17=1.1764 \ldots
$$

and C.-H. Jia [8] improved this to

$$
1<c<13 / 11=1.1818 \ldots .
$$

In this paper we obtain a further improvement. We prove the following

THEOREM. Let $c$ be a fixed real number in the range

$$
1<c<45 / 38=1.1842 \ldots
$$

Then (1.2) holds with $\rho_{0}=1 / 20$.

REMARK. The main point in the proof is to replace the original Fouvry-Iwaniec estimate with its refined version due to H.-Q. Liu [14]. The constant 45/38 may be improved somewhat but the numerical work showed that one cannot make a serious progress without new exponential sum estimates (even the value 1.185 would require more complicated decomposition in Section 5 in order to obtain a positive lower bound).

Throughout the paper, we suppose that $13 / 11 \leq c<45 / 38$, and denote $\gamma=1 / c$; $\varepsilon>0$ is a sufficiently small fixed number depending at most on $c, \eta=\varepsilon^{2}, X>X_{0}(\varepsilon)$. 
The letters $p, q, r, s$ always denote prime numbers. The notations $m \sim M$ and $A \asymp B$ mean that $M<m \leq 2 M$ and $A \ll B \ll A$. We write $L=\log X, e(x)=\exp (2 \pi i x)$, $\mathcal{N}(n)=\left[-n^{\gamma}\right]-\left[-(n+1)^{\gamma}\right], \psi(t)=t-[t]-1 / 2, r(t)=\psi\left(-(t+1)^{\gamma}\right)-\psi\left(-t^{\gamma}\right)$.

The constants implied by $\ll$ and $\mathcal{O}(\cdot)$ notation depend at most on $c$ and $\varepsilon$.

2. Outline of the method. We write

$$
P(z)=\prod_{p<z} p
$$

and for any sequence of integers $\mathcal{E}$

$$
\mathcal{E}_{d}=\{n \in \mathcal{E}: d \mid n\}, \quad S(\mathcal{E}, z)=|\{n \in \mathcal{E}:(n, P(z))=1\}| .
$$

Let us define

$$
\mathcal{A}=\left\{n: n \sim X, \quad n=\left[m^{c}\right]\right\}, \quad \mathcal{B}=\{n: n \sim X\} .
$$

We have

$$
\sum_{X<p \leq 2 X} \mathcal{N}(p)=S\left(\mathcal{A},(2 X)^{\frac{1}{2}}\right)+\mathcal{O}(1) .
$$

Thus to prove the Theorem it is sufficient to show that

$$
S\left(\mathcal{A},(2 X)^{\frac{1}{2}}\right) \geq 0.051 \frac{\lambda X}{\log X},
$$

where $\lambda=X^{\gamma-1}\left(2^{\gamma}-1\right)$.

In order to prove (2.1) we use the Buchstab identity

$$
S\left(\mathcal{E}, z_{1}\right)=S\left(\mathcal{E}, z_{2}\right)-\sum_{z_{2} \leq p<z_{1}} S\left(\mathcal{E}_{p}, p\right)
$$

together with asymptotic formulae of the form

$$
\sum_{d \sim D} a(d) S\left(\mathcal{A}_{d}, z(d)\right)=\lambda \sum_{d \sim D} a(d) S\left(\mathcal{B}_{d}, z(d)\right)+\text { error terms }
$$

where $a(m) \geq 0$ and $D, z(d)$ are suitably chosen. Applying (2.2) several times we decompose $S\left(\mathcal{A},(2 X)^{\frac{1}{2}}\right)$ into sums of the form appearing in the left-hand side of (2.3). For some of them we obtain asymptotic formulae of the above type and the rest may be discarded.

The most troublesome error terms arising in the proof of the asymptotic formulae of the form (2.3) are sums of the type

$$
\sum_{m \sim M} \sum_{n \sim N} a(m) b(n) r(m n)
$$


Using the Fourier expansion of the function $\psi(\cdot)$ we reduce their estimation to the estimation of exponential sums of the form

$$
\sum_{h \sim H} \sum_{m \sim M} \sum_{n \sim N} c(h) a(m) b(n) e\left(h(m n)^{\gamma}\right) .
$$

The estimates for these sums used before are good enough if the sizes of $H, M, N$ satisfy some conditions (see Section 3 for details) and $11 / 13<\gamma<1$. It was the last condition that set the limit of the method in [8]. We can replace it with the less restrictive $16 / 19<\gamma<1$, which allows us to obtain the better result.

3. Exponential sums. In this section we prove the exponential sum estimates which we need. Lemma 1 is proved by D. R. Heath-Brown [6, Lemma 4]. Lemma 2 is Theorem 3 of H.-Q. Liu [14]; it contains an improved version of Theorem 3 of [2] and Lemma 1 of [1]. Lemmas 3 and 4 are refined versions of Lemmas 4 and 5 of [1] and Lemmas 1 and 2 of [8].

Lemma 1. Let $5 / 6+\eta \leq \gamma<1, M N \asymp X, H \leq X^{1-\gamma+4 \eta}$. Assume further that $a(m)$, $b(n), c(h)$ are complex numbers of modulus $\leq 1$, and $N$ satisfies one of the conditions

$$
X^{1-\gamma+\varepsilon}<N<X^{5 \gamma-4-\varepsilon}
$$

or

$$
X^{5-5 \gamma+\varepsilon}<N<X^{\gamma-\varepsilon}
$$

Then

$$
\sum_{h \sim H} \sum_{m \sim M} \sum_{n \sim N} c(h) a(m) b(n) e\left(h(m n)^{\gamma}\right) \ll X^{1-5 \eta} .
$$

Lemma 2. Let $H \geq 1, X \geq 1, Y \geq 1$; let $\alpha, \beta$ and $\gamma$ be real numbers such that $\alpha \gamma(\beta-1)(\gamma-1) \neq 0$, and $A>C(\alpha, \beta, \gamma)>0, f(h, x, y)=A h^{\alpha} x^{\beta} y^{\gamma}$. Define

$$
S(H, X, Y)=\sum_{h \sim H} \sum_{x \sim X} \sum_{y \sim Y} a(h, x) b(y) e(f(h, x, y)) .
$$

Also suppose that $|a,(h, x)| \leq 1,|b(y)| \leq 1, F=A H^{\alpha} X^{\beta} Y^{\gamma} \gg Y$. Then

$$
\begin{array}{r}
L^{-3} S(H, X, Y) \ll(H X)^{19 / 22} Y^{13 / 22} F^{3 / 22}+H X Y^{5 / 8}\left(1+Y^{7} F^{-4}\right)^{1 / 16}+ \\
(H X)^{29 / 32} Y^{7 / 8} F^{-1 / 16} M^{5 / 32}+(H X)^{3 / 4} Y M^{1 / 4}
\end{array}
$$

where $L=\log (A H X Y+2), M=\max \left(1, F Y^{-2}\right)$.

Lemma 3. Let $16 / 19+\varepsilon \leq \gamma<1, M N \asymp X, H \leq X^{1-\gamma+4 \eta}$. Assume further that $a(m)$, $b(n), c(h)$ are complex numbers of modulus $\leq 1$, and $N$ satisfies the condition

$$
X^{3-3 \gamma+\varepsilon}<N<X^{3 \gamma-2-\varepsilon} .
$$


Then

$$
\sum_{h \sim H} \sum_{m \sim M} \sum_{n \sim N} c(h) a(m) b(n) e\left(h(m n)^{\gamma}\right) \ll X^{1-5 \eta} .
$$

Proof. Let us suppose first that $N \leq X^{1 / 2}$. Then we apply Lemma 2 with $(h, x, y)=(h, n, m)$ and using the assumptions of the lemma obtain

$$
X^{-\eta} \sum_{h \sim H} \sum_{m \sim M} \sum_{n \sim N} c(h) a(m) b(n) e\left(h(m n)^{\gamma}\right) \ll X^{1-\varepsilon / 4} .
$$

For $N \geq X^{1 / 2}$ we interchange the roles of $m$ and $n$.

Lemma 4. Let $16 / 19+\varepsilon \leq \gamma<1, M N \asymp X, H \leq X^{1-\gamma+4 \eta}$. Assume further that $a(m)$, $c(h)$ are complex numbers of modulus $\leq 1$, and $N$ satisfies the condition

$$
N>X^{3-3 \gamma+\varepsilon}
$$

Then

$$
\sum_{h \sim H} \sum_{m \sim M} \sum_{n \sim N} c(h) a(m) e\left(h(m n)^{\gamma}\right) \ll X^{1-5 \eta} .
$$

Proof. If $N$ satisfies the condition (3.3), Lemma 4 is a consequence of Lemma 3. Let us suppose now that $N \geq X^{3 \gamma-2-\varepsilon}$. We first apply Poisson summation formula (Lemma 6 of D. R. Heath-Brown [6]) and obtain

$$
\begin{aligned}
& \sum_{h \sim H} \sum_{m \sim M} \sum_{m \sim N} c(h) a(m) e\left(h(m n)^{\gamma}\right) \\
= & N F^{-1 / 2} \sum_{h \sim H} \sum_{m \sim M} \sum_{k} c(h) a(m) b(k) e(f(h, m, k))+\mathcal{O}\left(H M\left(N F^{-1 / 2}+L\right)\right),
\end{aligned}
$$

where $F=H X^{\gamma}, f(h, m, k)=B \cdot\left(h m^{\gamma} k^{-\gamma}\right)^{1 /(1-\gamma)} \asymp F, a(m), b(k), c(h)$ are of modulus $\leq 1\left(a(m), c(h)\right.$ possibly not the same), and $k$ runs through an interval $\left[K_{1}, K_{2}\right]$ for which $K_{i} \asymp K=F N^{-1}$. Now we use a variant of the Perron formula (Lemma 6 of E. Fouvry and H. Iwaniec [2] or formula (4.4) below) to remove the dependence between the summation variables. We get

$$
\begin{aligned}
& \sum_{h \sim H} \sum_{m \sim M} \sum_{n \sim N} c(h) a(m) e\left(h(m n)^{\gamma}\right) \\
& \ll L N F^{-1 / 2}\left|\sum_{h \sim H} \sum_{m \sim M} \sum_{k \sim K} c(h) a(m) b(k) e(f(h, m, k))\right|+H M\left(N F^{-1 / 2}+L\right) .
\end{aligned}
$$

We estimate the last sum via Lemma 2 with $(h, x, y)=(h, m, k)$ and the required estimate follows immediately.

4. Asymptotic formulae. We begin this section with two lemmas concerning the so called Buchstab's function $w(u)$ which is defined to be the continuous solution of the differential-difference equation 


$$
\left\{\begin{array}{cl}
w(u)=1 / u, & \text { for } 1<u<2, \\
(u w(u))^{\prime}=w(u-1), & \text { for } u>2 .
\end{array}\right.
$$

Lemma 5 follows easily from Lemma 2 of J. B. Friedlander [3], and Lemma 6 follows from Lemma 5 by partial summation.

Lemma 5. Let $\varepsilon>0$ be fixed, $x \geq x_{0}(\varepsilon), x^{\varepsilon} \leq z \leq x$. Then for any $u \in(x, 2 x]$ we have

$$
\sum_{\substack{x<n \leq u \\(n, P(z))=1}} 1=w\left(\frac{\log x}{\log z}\right) \cdot \frac{u-x}{\log z}+\mathcal{O}\left(\frac{x}{\log ^{2} x}\right) .
$$

LEMma 6. Under the assumption of Lemma 5 we have

$$
\sum_{\substack{n \sim x \\(n, P(z))=1}} \gamma n^{\gamma-1}=\lambda \sum_{\substack{n \sim x \\(n, P(z))=1}} 1 \cdot\left(1+\mathcal{O}\left(\frac{1}{\log x}\right)\right) .
$$

with $\lambda=\left(2^{\gamma}-1\right) x^{\gamma-1}$.

Lemmas 7 through 10 provide us with the asymptotic formulae of the form (2.3) which we need in the next section.

LeMma 7. Let $16 / 19+\varepsilon \leq \gamma<1, M N \asymp X$, and $a(m), b(n)$ are complex numbers of modulus $\ll X^{\eta / 3}$. Assume further that $N$ satisfies one of the conditions (3.1)-(3.3). Then

$$
\sum_{\substack{m \sim M \\ m n \in \mathcal{A}}} \sum_{\substack{n \sim N \\ m}} a(m) b(n)=\sum_{\substack{m \sim M \\ m n \in \mathcal{B}}} \sum_{\substack{n \sim N \\ m n}} a(m) b(n) \gamma(m n)^{\gamma-1}+\mathcal{O}\left(X^{\gamma-3 \eta}\right) .
$$

Moreover, if $N$ satisfies (3.4) then

$$
\sum_{\substack{m \sim M \\ m n \in \mathcal{A}}} \sum_{\substack{n \sim N \\ m}} a(m)=\sum_{\substack{m \sim M \\ m n \in \mathcal{B}}} \sum_{\substack{n \sim N \\ m n}} a(m) \gamma(m n)^{\gamma-1}+\mathcal{O}\left(X^{\gamma-3 \eta}\right) .
$$

Proof. Let us consider (4.1). We have

$$
\sum_{\substack{m \sim M \\ m n \in \mathcal{A}}} \sum_{\substack{n \sim N \\ m}} a(m) b(n)=\sum_{\substack{m \sim M \\ m n \in \mathcal{B}}} \sum_{\substack{n \sim N \\ m \in}} a(m) b(n) \mathcal{N}(m n)
$$

and therefore

$$
\sum_{\substack{m \sim M \\ m n \in \mathcal{A}}} \sum_{\substack{n \sim N \\ m}} a(m) b(n)=\Sigma_{1}+\Sigma_{2}
$$

where 


$$
\begin{aligned}
& \sum_{1}=\sum_{\substack { m \sim M \\
\begin{subarray}{c}{m \\
m \in \mathcal{B}{ m \sim M \\
\begin{subarray} { c } { m \\
m \in \mathcal { B } } }\end{subarray}} a(m) b(n)\left((m n+1)^{\gamma}-(m n)^{\gamma}\right) . \\
& \sum_{2}=\sum_{\substack{m \sim M \\
m n \in \mathcal{B}}} \sum_{\substack{n \sim N \\
m}} a(m) b(n) r(m n) .
\end{aligned}
$$

It is easy to see that

$$
\sum_{1}=\sum_{\substack{m \sim M \\ m n \in \mathcal{B}}} \sum_{n \sim N} a(m) b(n) \gamma(m n)^{\gamma-1}+\mathcal{O}\left(X^{\eta}\right) .
$$

Thus the proof of (4.1) will be completed if we show that

$$
\sum_{2} \ll X^{\gamma-3 \eta}
$$

Applying a well-known reduction argument using the Fourier expansion of the function $\psi(t)$ (see [6, pp. 245-247]) we find that the last inequality follows from the estimate

$$
\sum_{h \sim H} \sum_{m \sim M} \sum_{n \sim N} c(h) a(m) b(n) e\left(h(m n)^{\gamma}\right) \ll X^{1-5 \eta}
$$

where $H \leq X^{1-\gamma+4 \eta}$ and the coefficients $a(m), b(n), c(h)$ are complex numbers of modulus $\leq 1$. This estimate is provided by Lemma 1 if $N$ satisfies (3.1) or (3.2), and by Lemma 3 if $N$ satisfies (3.3).

One can prove (4.2) similarly using Lemma 4 instead of Lemmas 1 and 3.

Lemma 8. Let $16 / 19+\varepsilon \leq \gamma<1, M N \asymp X$ and $N$ satisfies one of the conditions (3.1)-(3.3). Let $I, J$ are integers and $\mathcal{I}_{i}, \mathcal{J}_{j}$ are intervals for $1 \leq i \leq I, 1 \leq j \leq J$. Write

$$
a(m, n)=\sum_{\substack{k p_{1} \cdots p_{I}=n \\ p_{1}<p_{2}<\cdots<p_{I} \\ p_{i} \in \mathcal{I}_{i}}} c(n) \sum_{\substack{l q_{1} \cdots q_{J}=m \\ q_{1}<q_{2}<\cdots<q_{J} \\ q_{j} \in \mathcal{J}_{j}}} d(m)
$$

with $|c(n)|,|d(m)| \leq 1$ and $p_{1}, \ldots, p_{I}$ and $q_{1}, \ldots, q_{J}$ satisfying $t$ joint conditions of the form

$$
p_{u} \leq q_{v} \quad \text { or } \quad q_{v} \leq p_{u}
$$

or

$$
\prod_{u \in \mathcal{U}} p_{u} \prod_{v \in \mathcal{V}} q_{v} \leq H \quad \text { or } \quad \prod_{u \in \mathcal{U}} p_{u} \geq \prod_{v \in \mathcal{V}} q_{v}
$$

or similar (for given $\mathcal{U} \subset\{1, \ldots, I\}, \mathcal{V} \subset\{1, \ldots, J\}, H \leq X)$. Then

$$
\sum_{\substack{m \sim M \\ m n \in \mathcal{A}}} \sum_{\substack{n \sim N\\}} a(m, n)=\sum_{\substack{m \sim M \\ m n \in \mathcal{B}}} \sum_{\substack{n \sim N \\ m n}} a(m, n) \gamma(m n)^{\gamma-1}+\mathcal{O}\left(X^{\gamma-3 \eta} L^{t}\right) .
$$


Proof. Each joint condition can be removed by the truncated Perron formula

$$
\frac{1}{\pi} \int_{-T}^{T} e^{i y \alpha} \frac{(\sin y \beta)}{y} d y=\left\{\begin{array}{cc}
1+\mathcal{O}\left(T^{-1}(\beta-|\alpha|)^{-1}\right), & \text { if }|\alpha| \leq \beta \\
\mathcal{O}\left(T^{-1}(|\alpha|-\beta)^{-1}\right), & \text { if }|\alpha|>\beta
\end{array}\right.
$$

at the cost of an additional $L$ factor in the error term.

For example, in order to remove the condition $p_{u} \leq q_{v}$ we apply (4.4) with $\alpha=\log p_{u}, \beta=\log \left(q_{v}+\frac{1}{2}\right)$ and $T=X^{2}$. We find

$$
\sum_{\substack{m \sim M \\ m n \in \mathcal{A}}} \sum_{\substack{n \sim N \\ m \in \mathcal{A}}} a(m, n)=\frac{1}{\pi} \int_{-T}^{T} \sum_{\substack{m \sim M \\ m n \in \mathcal{A}}} \sum_{\substack{n \sim N \\ m n}} a_{1}(m, n, y) \frac{d y}{y}+\mathcal{O}(1)
$$

where

$$
a_{1}(m, n, y)=a_{1}(m, n) p_{u}^{i y} \sin \left(y \log \left(q_{v}+\frac{1}{2}\right)\right)
$$

and $a_{1}(m, n)$ is the same as $a(m, n)$ but with the condition $p_{u} \leq q_{v}$ removed. Applying this procedure $t$ times we obtain

$$
\sum_{\substack{m \sim M \\ m n \in \mathcal{A}}} \sum_{\substack{n \sim N \\ \pi^{t}}} a(m, n)=\frac{1}{\pi^{t}} \int_{-T}^{T} \ldots \int_{-T}^{T} \sum_{\substack{m \sim M \\ m n \in \mathcal{A}}} \sum_{\substack{n \sim N \\ m}} \frac{a^{*}(m, n, \mathbf{y})}{y_{1} \ldots y_{t}} d \mathbf{y}+\mathcal{O}(1)
$$

where $a^{*}(m, n, \mathbf{y})$ is defined similarly to $a(m, n)$ but with all the joint conditions removed, so that

$$
a^{*}(m, n, \mathbf{y})=a(m, \mathbf{y}) b(n, \mathbf{y}) .
$$

We can therefore apply (4.1) to the last sum. We get

$$
\begin{aligned}
& \frac{1}{\pi^{t}} \int_{-T}^{T} \cdots \int_{-T}^{T} \sum_{\substack{m \sim M \\
m n \in \mathcal{A}}} \sum_{\substack{n \sim N \\
m n}} \frac{a(m, \mathbf{y}) b(n, \mathbf{y})}{y_{1} \cdots y_{t}} d \mathbf{y} \\
= & \frac{1}{\pi^{t}} \int_{-T}^{T} \cdots \int_{-T}^{T} \sum_{\substack{m \sim M \\
m n \in \mathcal{B}}} \sum_{\substack{n \sim N \\
m \in(m, \mathbf{y}) b(n, \mathbf{y})}} \frac{a(m n)^{\gamma-1} d \mathbf{y}+\mathcal{O}\left(X^{\gamma-3 \eta} L^{t}\right)}{y_{1} \cdots y_{t}} \gamma\left({ }^{2}\right)
\end{aligned}
$$

Applying (4.4) $t$ more times we finally find

$$
\begin{aligned}
& \frac{1}{\pi^{t}} \int_{-T}^{T} \cdots \int_{-T}^{T} \sum_{\substack{m \sim M \\
m n \in \mathcal{B}}} \sum_{\substack{n \sim N \\
m n \in}} \frac{a(m, \mathbf{y}) b(n, \mathbf{y})}{y_{1} \cdots y_{t}} \gamma(m n)^{\gamma-1} d \mathbf{y} \\
= & \sum_{\substack{m \sim M \\
m n \in \mathcal{B}}} \sum_{\substack{n \sim N\\
}} a(m, n) \gamma(m n)^{\gamma-1}+\mathcal{O}(1) .
\end{aligned}
$$


The lemma follows from (4.5)-(4.7).

Lemma 9. Let $u \geq 1$ and for some $N$ satisfying one of the conditions (3.1)-(3.3) there exists $\mathcal{D} \subset\{1, \ldots, u\}$ such that

$$
\prod_{k \in \mathcal{D}} p_{k} \sim N
$$

Then

$$
\sum_{p_{1}, \ldots, p_{u}} S\left(\mathcal{A}_{p_{1}} \ldots p_{u}, p_{1}\right)=\lambda \sum_{p_{1}, \ldots, p_{u}} S\left(\mathcal{B}_{p_{1}} \ldots p_{u}, p_{1}\right)\left(1+\mathcal{O}\left(L^{-1}\right)\right)+\mathcal{O}\left(X^{\gamma-\eta}\right)
$$

Here the summation is over prime numbers $p_{1}, \ldots, p_{u} \geq X^{1 / 15}$ satisfying $p_{k}>p_{1}$, together with $\ll 1$ further conditions of the type

$$
p_{k} \leq p_{l} \text { or } Q \leq \prod_{k \in \mathcal{F}} p_{k} \leq R
$$

(for some $\mathcal{F} \subset\{1, \ldots, u\}$ and $R \leq X)$.

Proof. The left-hand side of (4.8) equals

$$
\sum_{p_{1}, \ldots, p_{u}} \sum_{\substack{k: \\ p_{1} \ldots p_{u} k \in \mathcal{A} \\\left(k, P\left(p_{1}\right)\right)=1}} 1
$$

We can write this sum in the form

$$
\sum_{\substack{m \sim M \\ m n \in \mathcal{A}}} \sum_{\substack{n \sim N \\ m}} a(m, n)
$$

where

$$
n=\prod_{j \in \mathcal{D}} p_{j}, \quad m=\left(\sum_{j \notin \mathcal{D}} p_{j}\right) \cdot k
$$

and $a(m, n)$ are of the form considered in Lemma 8 . Since $N$ satisfies one of the conditions (3.1)-(3.3), we can use Lemma 8 for the last sum, so that we obtain

$$
\begin{aligned}
& \sum_{p_{1}, \ldots, p_{u}} S\left(\mathcal{A}_{p_{1}} \ldots, p_{u}, p_{1}\right) \\
= & \sum_{\substack{m \sim M \\
m n \in \mathcal{A}}} \sum_{p_{1}, \ldots, p_{u}} a(m, n) \gamma(m n)^{\gamma-1}+\mathcal{O}\left(X^{\gamma-3 \eta} L^{a}\right) \\
= & \sum_{\substack{k: \\
p_{1} \ldots p_{u} k \in \mathcal{B} \\
\left(k, P\left(p_{1}\right)\right)=1}} \gamma\left(p_{1} \cdots p_{u} k\right)^{\gamma-1}+\mathcal{O}\left(X^{\gamma-\eta}\right) .
\end{aligned}
$$


Here $a$ is the number of the joint conditions between the variables.

Applying Lemma 6 to the inner sum we complete the proof of the lemma.

Lemma 10. Suppose that $\gamma \geq 38 / 45+\varepsilon$ and $M \leq X^{8 / 15}$. Suppose further that $a(m)$ are real numbers, such that $0 \leq a(m) \ll X^{\eta}$ and $a(m)=0$ unless $p \mid m \Rightarrow p \geq X^{1 / 15}$. Then we have

$$
\sum_{m \sim M} a(m) S\left(\mathcal{A}_{m}, X^{1 / 15}\right)=\lambda \sum_{m \sim M} a(m) S\left(\mathcal{B}_{m}, X^{1 / 15}\right)\left(1+\mathcal{O}\left(L^{-1}\right)\right)+\mathcal{O}\left(X^{\gamma-\eta}\right) .
$$

Proof. We follow the approach of G. Harman [5, Lemma 2]. We shall make use of the Eratosthenes-Legendre sieve, which states that

$$
\sum_{\substack{n \leq N \\(n, P(z))=1}} f(n)=\sum_{\substack{d \mid P(z) \\ n d \leq N}} \mu(d) f(n d)
$$

where $\mu(d)$ is the Möbius function.

We take $z=X^{1 / 15}$ and applying (4.9) to $S\left(\mathcal{A}_{m}, z\right)$ we find

$$
\sum_{m \sim M} a(m) S\left(\mathcal{A}_{m}, z\right)=\sum_{m \sim M} a(m) \sum_{\substack{d \mid P(z) \\ m n d \in \mathcal{A}}} \mu(d) .
$$

Now we proceed to show that

$$
\sum_{m \sim M} a(m) \sum_{\substack{d \mid P(z) \\ m n d \in \mathcal{A}}} \mu(d)=\sum_{m \sim M} a(m) \sum_{\substack{d \mid P(z) \\ m n d \in \mathcal{B}}} \mu(d) \gamma(m n d)^{\gamma-1}+\mathcal{O}\left(x^{\gamma-\eta}\right) .
$$

We first consider the case $M \geq X^{7 / 15}$. We produce a new variable $k=d n$ and find

$$
\sum_{m \sim M} a(m) \sum_{\substack{d \mid P(z) \\ m n d \in \mathcal{A}}} \mu(d)=\sum_{\substack{m \sim M \\ m k \in \mathcal{A}}} a(m) b(k)
$$

with $|b(k)| \leq \tau(k)(\tau(k)$ denotes the number of the positive divisors of the integer $k)$. Splitting up the values of $k$ into intervals of the form $(K, 2 K]$ we derive (4.11) from (4.1) with $(m, n)=(m, k)$.

Now suppose that $M \leq X^{7 / 15}$. We divide the sum in the left-hand side of (4.11) into two parts

$$
\begin{aligned}
& \sum_{1}=\sum_{m \sim M} a(m) \sum_{\substack{d \mid P(z) \\
m n d \in \mathcal{A} \\
m d \leq X^{8 / 15}}} \mu(d), \\
& \sum_{2}=\sum_{m \sim M} a(m) \sum_{\substack{d \mid P(z) \\
m n d \in \mathcal{A} \\
m d>X^{8 / 15}}} \mu(d) .
\end{aligned}
$$


In $\sum_{1}$ we produce a new variable $k=m d$ and get

$$
\sum_{1}=\sum_{\substack{k \leq X^{8 / 15} \\ k n \in \mathcal{A}}} b(k)
$$

where $|b(k)| \ll k^{\eta} \tau(k)$. Therefore (4.2) with $(m, n)=(k, n)$ implies

$$
\sum_{1}=\sum_{m \sim M} a(m) \sum_{\substack{d \mid P(z) \\ m n d \in \mathcal{B} \\ m d \leq X^{8 / 15}}} \mu(d) \gamma(m n d)^{\gamma-1}+\mathcal{O}\left(X^{\gamma-2 \eta}\right) .
$$

Now we write $\sum_{2}$ in the form

$$
\sum_{2}=-\sum_{m \sim M} a(m) \sum_{p<z} \sum_{\substack{d \mid P(p) \\ m n p d \in \mathcal{A} \\ m p d>X^{8 / 15}}} \mu(d)
$$

and divide the last sum into two parts

$$
\begin{gathered}
\sum_{3}=\sum_{m \sim M} a(m) \sum_{p<z} \sum_{\substack{d \mid P(p) \\
m n p d \in \mathcal{A} \\
m d \leq X^{8 / 15}<m p d}} \mu(d), \\
\sum_{4}=\sum_{m \sim M} a(m) \sum_{p<z} \sum_{\substack{d \mid P(p) \\
m n p d \in \mathcal{A} \\
m d>X^{8 / 15}}} \mu(d) .
\end{gathered}
$$

In $\sum_{3}$ we produce two new variables $k=m d$ and $l=n p$ and we get (note that $p<z, m p d>X^{8 / 15} \Rightarrow k>X^{7 / 15}$ )

$$
\sum_{3}=\sum_{\substack{X^{7 / 15}<k \leq X^{8 / 15} \\ k l \in \mathcal{A}}} b(k, l)
$$

where $|b(k, l)| \ll k^{\eta} \tau(k) \tau(l)$ is of the form appearing in the left-hand side of (4.3) with the only two joint conditions

$$
p>q \text { and } p k>X^{8 / 15}
$$

(here $q$ is the largest prime divisor of $d$ ). Therefore Lemma 8 with $(m, n)=(k, l)$ implies

$$
\sum_{3}=\sum_{m \sim M} a(m) \sum_{p<z} \sum_{\substack{d \mid P(p) \\ m n p d \in \mathcal{B} \\ m d \leq X^{8 / 15}<m p d}} \mu(d) \gamma(m n p d)^{\gamma-1}+\mathcal{O}\left(X^{\gamma-2 \eta} L^{2}\right)
$$

We treat $\sum_{4}$ similarly to $\sum_{2}$ and write it as the sum of two sums 


$$
\begin{gathered}
\sum_{5}=\sum_{m \sim M} a(m) \sum_{p_{2}<p_{1}<z} \sum_{\substack{d \mid P\left(p_{2}\right) \\
m n p_{1} p_{2} d \in \mathcal{A} \\
m d \leq X^{8 / 15}<m p d}} \mu(d), \\
\sum_{6}=\sum_{m \sim M} a(m) \sum_{p_{2}<p_{1}<z} \sum_{\begin{array}{c}
d \mid P\left(p_{2}\right) \\
m n p_{1} p_{2} d \in \mathcal{A} \\
m d>X^{8 / 15}
\end{array}} \mu(d) .
\end{gathered}
$$

We deal with $\sum_{5}$ as we did with $\sum_{3}$ and obtain a similar asymptotic formula, and we give further decomposition for $\sum_{6}$. We can continue in this fashion obtaining each time $\sum_{2 j-1}$ for which we can apply Lemma 8 and $\sum_{2 j}$ for which we give further decomposition. Since the integers in the interval $(X, 2 X]$ have $<L$ prime divisors after at most $L$ such steps we will obtain an empty $\sum_{2 j}$ and we will have given asymptotic formulae for all the occurring sums. Clearly, combining the asymptotic formulae for all $\sum_{2 j-1}$ we complete the proof of (4.11) for $M<X^{7 / 15}$.

Applying (4.9) and Lemma 6 we find

$$
\sum_{m \sim M} a(m) \sum_{\substack{d \mid P(z) \\ m n d \in \mathcal{B}}} \mu(d) \gamma(m n d)^{\gamma-1}=\sum_{m \sim M} a(m) S\left(\mathcal{B}_{m}, z\right)\left(1+\mathcal{O}\left(L^{-1}\right)\right) .
$$

The lemma follows from (4.10)-(4.12).

5. Proof of the Theorem. We apply twice (2.2) and get

$$
\begin{aligned}
& S\left(\mathcal{A},(2 X)^{1 / 2}\right)=S\left(\mathcal{A}, X^{7 / 45}\right)-\sum_{X^{7 / 45} \leq p \leq X^{2 / 9}} S\left(\mathcal{A}_{p}, p\right) \\
& -\sum_{X^{7 / 15} \leq p \leq(2 X)^{1 / 2}} S\left(\mathcal{A}_{p}, p\right) \\
& -\sum_{X^{2 / 9}<p<X^{7 / 15}} S\left(\mathcal{A}_{p}, X^{1 / 15}\right) \\
& +\sum_{\substack{X^{2 / 9}<p<X^{7 / 15} \\
X^{1 / 15} \leq q<X^{7 / 45}}} S\left(\mathcal{A}_{p q}, q\right) \\
& +\sum_{\substack{X^{2 / 9}<p<X^{7 / 15} \\
X^{7 / 45} \leq q \leq X^{2 / 9}}} S\left(\mathcal{A}_{p q}, q\right) \\
& +\sum_{\substack{X^{2 / 9}<q<p<X^{7 / 15} \\
p q^{2} \leq 2 X}} S\left(\mathcal{A}_{p q}, q\right) \\
& =S_{1}-S_{2}-S_{3}-S_{4}+S_{5}+S_{6}+S_{7} \text {, say. }
\end{aligned}
$$

For $S_{1}, S_{5}$ and $S_{7}$ we give further decompositions. We apply to $S_{1}$ the Buchstab identity (2.2) four times and we get 


$$
\begin{aligned}
S_{1}=S\left(\mathcal{A}, X^{1 / 15}\right) & -\sum_{X^{1 / 15} \leq p<X^{7 / 45}} S\left(\mathcal{A}_{p}, X^{1 / 15}\right) \\
& +\sum_{X^{1 / 15} \leq q<p<X^{7 / 45}} S\left(\mathcal{A}_{p q}, X^{1 / 15}\right) \\
& -\sum_{X^{1 / 15} \leq r<q<p<X^{7 / 45}} S\left(\mathcal{A}_{p q r}, X^{1 / 15}\right) \\
& +\sum_{X^{1 / 15} \leq s<r<q<p<X^{7 / 45}} S\left(\mathcal{A}_{p q r s}, s\right) \\
& =S_{8}-S_{9}+S_{10}-S_{11}+S_{12}, \quad \text { say. }
\end{aligned}
$$

Now we consider $S_{5}$. We write $\left(\nabla_{1}\right)$ for the conditions

$$
X^{2 / 9}<p<X^{7 / 15}, \quad X^{1 / 15} \leq q<X^{7 / 45}, \quad p q^{2} \leq X^{8 / 15},
$$

and $\left(\nabla_{2}\right)$ for the conditions

$$
X^{2 / 9}<p<X^{7 / 15}, \quad X^{1 / 15} \leq q<X^{7 / 45}, \quad p q^{2}>X^{8 / 15} .
$$

We apply (2.2) two more times to the part of $S_{5}$ corresponding to the conditions $\left(\nabla_{1}\right)$ and get

$$
\begin{aligned}
\sum_{p, q:\left(\nabla_{1}\right)} S\left(\mathcal{A}_{p q}, q\right) & =\sum_{p, q:\left(\nabla_{1}\right)} S\left(\mathcal{A}_{p q}, X^{1 / 15}\right) \\
& -\sum_{\substack{p, q:\left(\nabla_{1}\right) \\
X^{1 / 15} \leq r<q}} S\left(\mathcal{A}_{p q r}, X^{1 / 15}\right) \\
& +\sum_{\substack{p, q:\left(\nabla_{1}\right) \\
X^{1 / 15} \leq s<r<q}} S\left(\mathcal{A}_{p q r s}, S\right) \\
& =S_{13}-S_{14}+S_{15}, \text { say. }
\end{aligned}
$$

For the part of $S_{5}$ corresponding to the conditions $\left(\nabla_{2}\right)$ we find

$$
\sum_{p, q:\left(\nabla_{2}\right)} S\left(\mathcal{A}_{p q}, q\right)=\sum_{p, q:\left(\nabla_{2}\right)} \sum_{\substack{n: \\ p q n \in \mathcal{A} \\(n, P(q))=1}} 1=S_{16}+S_{17}
$$

where

$$
\begin{aligned}
& S_{16}=\sum_{p, q:\left(\nabla_{2}\right)} \sum_{\substack{r: \\
p q r \in \mathcal{A} \\
r \geq q}} 1 \\
& S_{17}=\sum_{\substack{p, q,:\left(\nabla_{2}\right) \\
q \leq r \leq \sqrt{2 X / p q} \\
r \leq s \leq(2 X) / p q r}} \sum_{\substack{n: \\
p q r s n \in \mathcal{A} \\
(n, P(s))=1}} 1 .
\end{aligned}
$$

For $S_{7}$ we have 


$$
\begin{aligned}
S_{7} & =\sum_{\substack{X^{2 / 9}<q<p<X^{7 / 15} \\
p q<X^{7 / 15}}} S\left(\mathcal{A}_{p q}, q\right) \\
+ & \sum_{\substack{X^{2 / 9}<q<p<X^{7 / 15} \\
X^{7 / 15} \leq p q \leq X^{8 / 15}}} S\left(\mathcal{A}_{p q}, q\right) \\
+ & \sum_{\substack{X^{2 / 9}<q<p<X^{7 / 15} \\
X^{8 / 15}<p q<p q^{2} \leq 2 X}} S\left(\mathcal{A}_{p q}, q\right) \\
= & S_{18}+S_{19}+S_{20}, \quad \text { say. }
\end{aligned}
$$

Using the definition of the sifting function we obtain

$$
\begin{aligned}
S_{18} & =\sum_{\substack{X^{2 / 9}<q<p<X^{7 / 15} \\
p q<X^{7 / 15}}} \sum_{\substack{n: \\
p q n \in \mathcal{A} \\
(n, P(q))=1}} 1 \\
& =S_{21}+S_{22},
\end{aligned}
$$

where $S_{21}$ is the number of the integers of the form $p q r \in \mathcal{A}$ for which

$$
X^{2 / 9}<q<p<p q<X^{7 / 15} \text { and } r \geq q
$$

and $S_{22}$-the number of the integers of the form pqrs $\in \mathcal{A}$ for which

$$
X^{2 / 9}<q<p<p q<X^{7 / 15} \text { and } q \leq r \leq s .
$$

Hence,

$$
S_{7}=S_{19}+S_{20}+S_{21}+S_{22}
$$

Combining (5.1)-(5.4) and (5.7) we obtain

$$
\begin{aligned}
S\left(\mathcal{A},(2 X)^{1 / 2}\right)= & -S_{2}-S_{3}-S_{4}+S_{6}+S_{8}-S_{9}+S_{10}-S_{11}+S_{12}+S_{13} \\
& -S_{14}+S_{15}+S_{16}+S_{17}+S_{19}+S_{20}+S_{21}+S_{22} .
\end{aligned}
$$

Lemma 9 provides asymptotic formulae for $S_{2}, S_{3}, S_{6}$ and $S_{19}$, and Lemma 10 -for $S_{4}, S_{8}-S_{11}, S_{13}$ and $S_{14}$. Using Lemmas 8 and 9 we can also find asymptotic formulae for those parts of $S_{12}, S_{15}-S_{17}, S_{21}$ and $S_{22}$ where some subproduct of $p q r$ or pqrs lies in one of the intervals

$$
\left[X^{7 / 45}, X^{2 / 9}\right],\left[X^{7 / 15}, X^{8 / 15}\right],\left[X^{7 / 9}, X^{38 / 45}\right] .
$$

Since $S_{20}$ and the remaining parts of these sums give positive contribution to the right-hand side of (5.8), we may discard them obtaining a lower bound for $S\left(\mathcal{A},(2 X)^{1 / 2}\right)$. Now using the corresponding decomposition for $S\left(\mathcal{B},(2 X)^{1 / 2}\right)$ we obtain that

$$
S\left(\mathcal{A},(2 X)^{1 / 2}\right) \geq \lambda\left(S\left(\mathcal{B},(2 X)^{1 / 2}\right)-T_{12}-T_{15}-T_{16}-T_{17}-T_{20}-T_{21}-T_{22}\right) .
$$


Here $T_{12}, \ldots, T_{22}$ are almost the same as the discarded sums with the only difference that $\mathcal{A}$ is replaced by $\mathcal{B}$, for example,

$$
T_{20}=\sum_{\substack{X^{2 / 9}<q<p<X^{7 / 15} \\ X^{8 / 15}<p q<p q^{2} \leq 2 X}} S\left(\mathcal{B}_{p q}, q\right) .
$$

The Prime Number Theorem implies

$$
S\left(\mathcal{B},(2 X)^{1 / 2}\right)=\frac{X}{L}\left(1+\mathcal{O}\left(L^{-1}\right)\right)
$$

In order to evaluate $T_{12}, \ldots T_{22}$ we use Lemma 5 and the estimates for the Buchstab function contained in the following lemma (cf. Lemma 8 of [9]).

LEMma 11. If $w(x)$ is the Buchstab function, then we have

(a) $w(u) \leq 0.5644$ for $u \geq 3$;

(b) $w(u) \leq 0.5672$ for $u \geq 2$. obtain

We consider first $T_{20}$. Applying Lemma 5 and the Prime Number Theorem we

$$
\begin{aligned}
T_{20} & =\sum_{\substack{X^{2 / 9}<q<p<X^{7 / 15} \\
X^{8 / 15}<p q<p q^{2} \leq 2 X}} \frac{X}{p q \log q} w\left(\frac{\log (X / p q)}{\log q}\right)\left(1+\mathcal{O}\left(L^{-1}\right)\right) \\
& =\left(I_{20}+\mathcal{O}\left(L^{-1}\right)\right) \frac{X}{L}
\end{aligned}
$$

where

$$
I_{20}=\iint_{\mathcal{D}_{20}} \frac{1}{x y^{2}} w\left(\frac{1-x-y}{y}\right) d x d y
$$

and $\mathcal{D}_{20}$ is the region defined by the conditions

$$
2 / 9<y<x<7 / 15, \quad x+y>8 / 15, \quad x+2 y<1 .
$$

The computation of the last integral shows that

$$
T_{20} \leq 0.41695 \frac{X}{L}
$$

Now we consider $T_{21}$. We have 


$$
\begin{aligned}
T_{21} & \leq \sum_{\substack{X^{2 / 9}<q<p<X^{7 / 15} \\
p q<X^{7 / 15}}}\left(\pi\left(\frac{2 X}{p q}\right)-\pi\left(\frac{X}{p q}\right)\right) \\
& =\sum_{\substack{X^{2 / 9}<q<p<X^{7 / 15} \\
p q<X^{7 / 15}}} \frac{X}{p q \log (X / p q)}\left(1+\mathcal{O}\left(L^{-1}\right)\right) \\
& =\left(I_{21}+\mathcal{O}\left(L^{-1}\right)\right) \frac{X}{L}
\end{aligned}
$$

where

$$
I_{21}=\iint_{\mathcal{D}_{21}} \frac{d x d y}{x y(1-x-y)}
$$

and $\mathcal{D}_{21}$ is the region defined by the inequalities

$$
2 / 9<y<x<x+y<7 / 15 \text {. }
$$

Hence

$$
T_{21} \leq 0.004333 \frac{X}{L}
$$

Treating $T_{16}$ and $T_{22}$ similarly we get

$$
T_{16} \leq 0.35301 \frac{X}{L} \text { and } T_{22} \leq 0.000244 \frac{X}{L} .
$$

The sums $T_{12}$ and $T_{15}$ may be estimated similarly to $T_{20}$ via Lemma 5 and the Prime Number Theorem. We find

$$
T_{12} \leq 0.006183 \frac{X}{L} \text { and } T_{15} \leq 0.000386 \frac{X}{L} .
$$

Finally we consider $T_{17}$. We divide it into two parts: $T_{23}$ where

$$
\sqrt{\frac{2 X}{p q r}}<s \leq \frac{2 X}{p q r}
$$

and $T_{24}$ where

$$
s \leq \sqrt{\frac{2 X}{p q r}}
$$

and we obtain

$$
T_{17}=T_{23}+T_{24}
$$


With $T_{23}$ we proceed similarly to $T_{21}$, and with $T_{24}$ - similarly to $T_{20}$. We get

$$
T_{23} \leq 0.115868 \frac{X}{L}
$$

and

$$
T_{24} \leq 0.020259 \frac{X}{L}
$$

Finally from (5.9)-(5.17) we derive

$$
S\left(\mathcal{A},(2 X)^{1 / 2}\right) \geq 0.08 \frac{\lambda X}{L} .
$$

Acknowledgements. The author would like to express his gratitude to Profs. D. I. Tolev and O. T. Trifonov for the regular attention to his work. He would also like to thank the referee for his valuable comments. In particular, the author followed his suggestions on the proof of Lemma 10 which allowed to shorten considerably Section 4.

\section{REFERENCES}

1. R. C. Baker, G. Harman and J. Rivat, Primes of the form $\left[n^{c}\right]$, J. Number Theory $\mathbf{5 0}$ (1995), 261-277.

2. E. Fouvry and H. Iwaniec, Exponential sums with monomials, J. Number Theory 33 (1989), 311-333.

3. J. B. Friedlander, Integers free from large and small primes, Proc. London Math. Soc. (3) 33 (1976), 565-576.

4. G. Harman, On the distribution of $\alpha$ p modulo one, J. London Math. Soc. (2) 27 (1983), 9-18.

5. G. Harman, On the distribution of $\alpha$ p modulo one II, Proc. London Math. Soc. (3) 72 (1996), 241-260.

6. D. R. Heath-Brown, The Piatetski-Shapiro prime number theorem, J. Number Theory 16 (1983), 242-266.

7. C.-H. Jia, On Piatetski-Shapiro prime number theorem, Chinese Ann. Math. 15B:1 (1994), 9-22.

8. C.-H. Jia, On Piatetski-Shapiro prime number theorem II, Science in China Ser. A 35 (1993), 913-922.

9. C.-H. Jia, On the Piatetski-Shapiro-Vinogradov theorem, Acta Arith. 73 (1995), 1-28.

10. G. A. Kolesnik, The distribution of primes in sequnces of the form $\left[n^{c}\right]$, Mat. Zametki 2 (1969), 117-128.

11. G. A. Kolesnik, Primes of the form $\left[n^{c}\right]$, Pacific J. Math. 118 (1988), 81-92.

12. D. Leitmann, Abschätzung trigonometrischer summen, J. Reine Angew. Math. 317 (1980), 209-219. $81-92$.

13. D. Leitmann and D. Wolke, Primzahlen der gestalt $[f(n)]$, Math. Z. 145 (1975),

14. H.-Q. Liu, On the number of abelian groups of a given order (supplement), Acta Arith. 64 (1993), 285-296. 
15. H.-Q. Liu and J. Rivat, On the Piatetski-Shapiro prime number theorem, Bull. London Math. Soc. 24 (1992), 143-147.

16. I. I. Piatetski-Shapiro, On the distribution of prime numbers in sequences of the form [f(n)], Mat. Sb. 33 (1953), 559-566.

17. J. Rivat, Autour d'un theorem de Piatetski-Shapiro, Thesis, Universite de Paris Sud, 1992. 